\title{
The Research on Management Information System Design for Construction Engineering Quality
}

\author{
WU Yuling ${ }^{1, \text { a }}$ \\ ${ }^{1}$ Chongqing water resources and electric engineering college, Chongqing Yongchuan 402160 , \\ China \\ aWuyuling1028@yeah.net
}

Keywords: Engineering Quality, Construction Project, Management Information System

\begin{abstract}
With the rapid development of Chinese construction enterprise, construction quality problems also occur frequently. Improve the quality of construction projects on the one hand, to improve the project investment benefit, environmental benefit and social benefit, on the other hand also to provide a good life and property safety protection. And effectively improve the quality of construction projects is the project quality management and control, among them, the construction stage of quality management in the whole project quality management plays a decisive role. Therefore, this article choose construction engineering project construction stage of quality management, has important practical value. The construction engineering quality management information system development is not only beneficial to improve the staff's working efficiency and data transmission rate, alleviate the pressure of work, and also improve the manageability and accuracy of the data.
\end{abstract}

\section{Introduction}

Along with the computer, communications, network for the rapid development of high and new technology, the wide application of information technology in social fields, in fact is the application of information system. Use information system to replace the original manual management mode, and reengineering its original business processes, all kinds of enterprises to become the enterprise in the fierce market competition strategy. Use information system, it can realize automation management process, reduce the transactional work load of management, accelerate the speed of information processing, improve the quality and utilization of information, agile reflect changes in consumer demand, so information system by reducing the cost, improve the work quality, shorten the delivery cycle of a product or service and to get higher interest, etc., fundamentally improve the market competition ability of the enterprise [1].

Given China's increasing investment to infrastructure and the vigorous development of the real estate industry, construction industry is booming, and the national requirements for quality management of the construction industry is becoming more and more high. Construction quality management departments at all levels are using various means to strengthen the management of construction quality [2]. Computer information management, as a kind of modern management methods are widely used in construction quality management work, become an indispensable part of the quality inspection process. The level of information management has become a measure of a region, an enterprise management level of important symbol. Chinese architecture in the field of information technology starts late, for the field construction engineering quality supervision management information system development process also belong to explore. For the exploration of information system development, demand and planning problem is a major cause of lead to rework and project delays. So the study of this kind of problem for the implementation of the construction engineering quality supervision management information system is of great realistic significance.

\section{System related key technologies}

B/S (Browser/Server) mode is a three or more layers of a distributed system architecture. The server contains a Web server, database server, application server, such as the structure shown in Figure 1. 
When in this mode, Web server Web server through a browser client requests to query the database server, Web server query data in the form of a hypertext document browser. B / S mode application system is a thin client, the client uses a browser software, hardware configuration do not ask, easy to manage and maintain the systems, software development, upgrades and maintenance on the server side, reducing development and maintenance work the system does not require the development of client software, web browser software can be downloaded for free from the Internet, free upgrades, system protection business investment, B / S mode uses the standard TCP/IP, HTTP protocol, can be combined with the company's existing network. Also has good scalability, can be connected directly intern. So B / s mode is easy to use, easy to maintain a high degree of information sharing is gradually replacing the C/S mode [3-4].

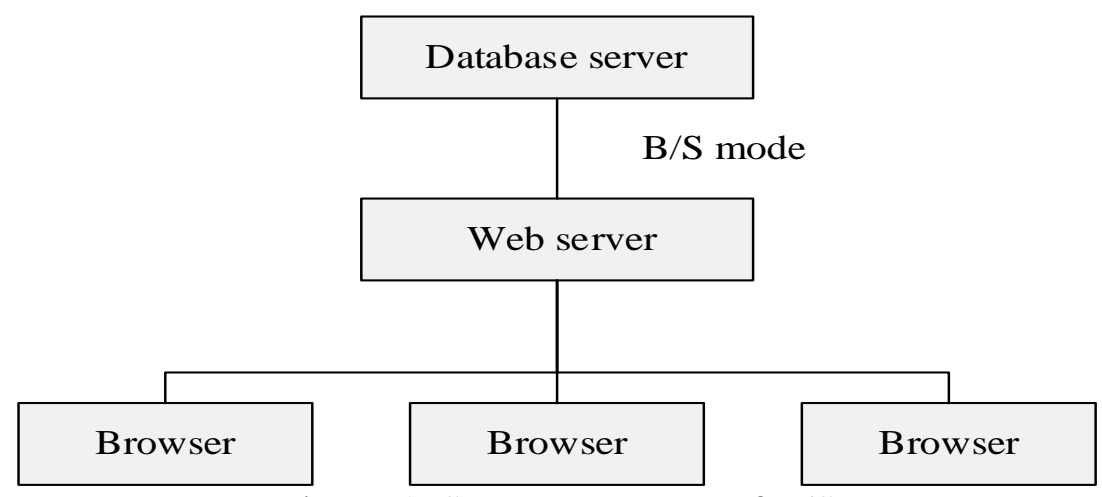

Figure 1. System structure of B/S

\section{Function analysis of the construction project quality management system}

Construction engineering quality management information system to the most modern technology and computer network management, and improve the company's overall economic effect, through the analysis of the company's organizational structure and related human resource allocation, sending the data related to management, and rapid response [5]. The system integration of enterprise management information system, provide for the management of high, medium and low class information, is mainly composed of people, computer and related facilities information system. In order to make the construction of the management system to achieve efficiency and practicability of the stronger, faster, must need a set of complete management information system, to achieve the standardization of construction safety management system, collectivization, the rules and regulations. Quality management system enables to obtain a higher economic interests of the company as a whole, in order to meet the current demand growing economy, and strengthen the competitiveness and vitality of a company in the market.

In the study of this paper, the construction project quality management system function module mainly includes the quality inspection, quality supervision, comprehensive management, and live video statistical query module, etc. Construction engineering construction quality management information system of user objects including construction engineering quality supervision departments, construction units and the quality detection unit, the quality department is responsible for the quality information data entry. Integrated management of user units' distribution, responsible for the supervision over the information input, processing and forming of personnel dedicated to the management mode, basic logic relationship between the various business units as shown in figure 3. 


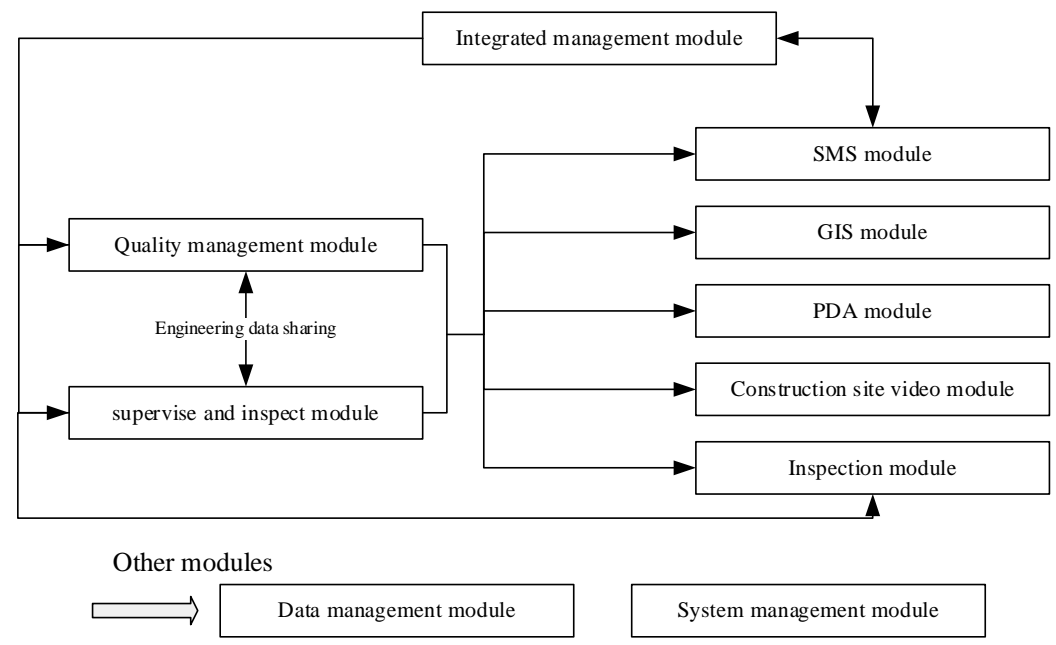

Figure 2.Function relationship of the construction project quality management system

\section{The design of construction project quality management system}

The construction process of quality management need to the road of informatization, simple administrative control measures is difficult to meet the requirements of construction market. In the construction project construction quality management information system, based on the use of modern computer and communication, effective implementation of the project quality management, to strengthen the supervision of construction engineering. This is currently the best solution in building engineering construction quality management. In addition, with the aid of construction quality management system, supervision units and enterprises quality management function gradually by the administrative institutions, shift to the service department. Quality supervision unit by the original administrative supervision, gradually development for information integration department, in order to realize the comprehensive monitoring of market and engineering. Based on the introduction to architecture system, based on $\mathrm{B} / \mathrm{S}$ structure of the construction quality information system platform structure is shown in figure 3.

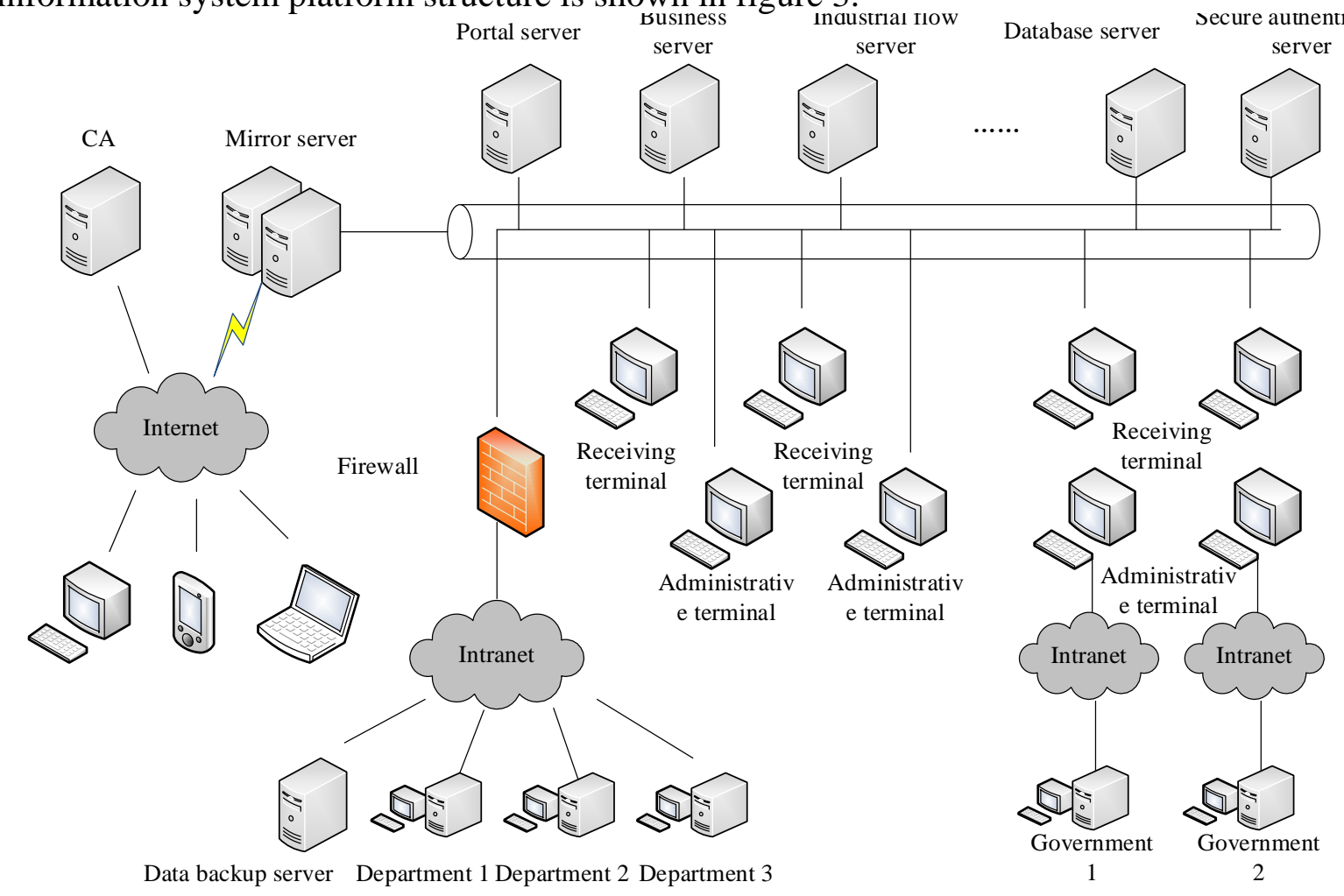

Figure 3.The structure of construction project quality management system

System access of users include different role or career, which involves project owners, units and regulators. After the user login system, can be directly for database access, and will also be able to 
read and write operations authorized by the browser. Other visitors are only in a Web browser to access the server. Web server can either with the database server in a single computer at the same time, can also be stored independently, communicate by ASP to realize interconnection between them.

This system design is the guiding ideology of all for the sake of users, the work interface makes every effort to clear and concise structure, through good organizational form of the interface, the user is easy to understand the various features, use, and to achieve the goal. A good system not only can let the staff from the cumbersome manual operation, and builders management information system is a kind of new mode of government supervision of construction market, as it further, the specification of the most efficient will drive construction market, and provide support for management and decision making. In order to meet this requirement, on the software implementation, this system to realize user authentication function, meet people of different identities set permissions, real-time dynamically show builders regulation, make the builders management more complete and standardize business processes, eliminate loopholes, reduce the workload of staff, improve the ability of handling affairs, improve work efficiency, and can provide strong information security, the user can communicate safely, comfortable office.

\section{Conclusion}

In this paper, it is based on the theory of demand management and planning, according to the characteristics of construction engineering quality supervision management, discusses the system requirements and the relationship between the implementation plan, and on the basis of demand analysis, using the function point count the scale of development, to develop the work breakdown structure, thus gives the critical path of the project, can provide guarantee for the smooth implement of the system. This paper for the quality of construction engineering project management informatization provides a complete set of implementation patterns, at present the system management model has been in the domestic several construction engineering management institution, and will be widely recognized by users. By introducing the system, the quality of construction engineering project management process was optimized, and get a great improve the efficiency of data management, to ensure the construction quality management standardization and fairness.

\section{References}

[1] R. Sacks, L. Koskela, and B.A. Dave: Journal of construction engineering and management, Vol. 136 (2010) No.9, p.968.

[2] L.D. Xu: International Journal of Production Research, Vol. 49 (2011) No.1, p.183.

[3] W. Shen, Q. Hao, and H. Mak: Advanced Engineering Informatics, Vol. 24 (2010) No.2, p.196.

[4] S.Y.L. Yin, H.P. Tserng, and J.C. Wang: Automation in Construction, Vol. 18 (2009) No.5, p.677.

[5] R. Sacks, M. Radosavljevic, and R. Barak: Automation in construction, Vol. 19 (2010) No.5, p.641. 